\title{
Tattoo Complaints and Complications: Diagnosis and Clinical Spectrum
}

\author{
Jørgen Serup • Katrina Hutton Carlsen • Mitra Sepehri \\ Department of Dermatology, 'Tattoo Clinic', Bispebjerg University Hospital, Copenhagen, Denmark
}

\begin{abstract}
Tattoos cause a broad range of clinical problems. Mild complaints, especially sensitivity to sun, are very common and seen in $1 / 5$ of cases. Medical complications are dominated by allergy to tattoo pigment haptens or haptens generated in the skin, especially in red tattoos but also in blue and green tattoos. Symptoms are major and can be compared to cumbersome pruritic skin diseases. Tattoo allergies and local reactions show distinct clinical manifestations, with plaque-like, excessive hyperkeratotic, ulcero-necrotic, lymphopathic, neuro-sensory, and scar patterns. Reactions in black tattoos are papulo-nodular and non-allergic and associated with the agglomeration of nanoparticulate carbon black. Tattoo complications include effects on general health conditions and complications in the psycho-social sphere. Tattoo infections with bacteria, especially staphylococci, which may be resistant to multiple antibiotics, may be prominent
\end{abstract}

and may progress into life-threatening sepsis. Contaminated tattoo ink is an open-window risk vector that can lead to epidemic tattoo infections across national borders due to contaminated bulk production. Hepatitis B and $C$ and human immunodeficiency virus (HIV) transferred by tattooing remain a significant risk needing active prevention. It is noteworthy that cancer arising in tattoos, in regional lymph nodes, and in other organs due to tattoo pigments and ingredients has not been detected or noted as a significant clinical problem hitherto, despite millions of people being tattooed for decennia. Clinical observation and epidemiology disagree with register data, which indicate an increased risk of cancer due to chemical carcinogens present in some inks. Registers rely on chronic dosaging of cell lines and animals. However, tattooing in humans is essentially a single-dose exposure, which might explain the observed discrepancy.

(c) 2015 S. Karger AG, Basel 


\section{Introduction}

Tattooing of skin via deposition of pigment particles and ink ingredients in the dermis changes normal skin into abnormal skin. Fortunately, this often causes no harm and no disease, although with important exceptions. The tattooed individual may risk disablement, and death may even occur under exceptional circumstances.

In the medical literature, case reports and reviews have outlined the wide spectrum of adverse events related to tattooing [1-9]. Reports should always be read in the context of the development of tattoo inks and the tattoo business at the time of the report. Inorganic pigments such as mercury and cadmium salts are now being replaced by organic pigments. The tattoo ink products of today remain composite, with unknown ingredients and a range of chemical contaminants. The bacterial contamination and uncertain sterility of inks are even challenges today $[10,11]$.

Tattoo inks contain soluble ingredients, which supposedly are distributed, metabolised, and excreted within a few days, and the pigment. Tattooing is a single-dose injection of a large local dose, depending on the size of the tattoo. However, robust pigment particles remain permanently in the skin and make up the desired colouring. Pigments in the skin slowly vanish over time, along with a slow release of minute amounts of chemicals and metabolites, which may actually cause harm, such as allergy. The release is triggered by enzymatic and cellular processes in the tissues that are very different from harsh chemical degradation, with the formation of chemical cleavage products, in the laboratory, which is an artificial and unrealistic scenario. Pigments instilled into the tattoo partly escape via the lymph and become deposited in the regional lymph nodes. The nodes are invisibly dyed along with the skin. However, unknown amounts of pigment nanoparticles might reach the blood stream and theoretically cause harm somewhere in the body.
It has been documented that many tattoo inks, as referenced to register data, contain carcinogenic, mutagenic, and reprotoxic substances. However, concerns about tattoos' induction of skin cancer, including malignant melanoma, have not been confirmed in the medical literature and remain hypothetical [12]. A systematic review of the world literature only detected 50 reports of skin cancers situated in tattoos, which was concluded to be a coincidence since spontaneous skin malignancy related to sun exposure is very common and may affect tattooed skin independent of the pigment [12]. The literature, including the cited reviews, has not indicated a risk of distant organ cancer or foetal hazards, although such risks have not been excluded. Bispebjerg University Hospital, Department of Dermatology includes a centre for cutaneous oncology. Neither 'Oncology' nor the 'Tattoo Clinic' noted cases in which the origin of skin cancer was attributed to a tattoo and its pigment. Clinical observation and epidemiology disagree with register data, which indicate an increased risk of cancer due to chemical carcinogens present in inks. Registers rely on chronic dosaging of cell lines and animals, however, tattooing in humans is essentially a singledose exposure, which might explain the observed discrepancy.

Since 2008, the 'Tattoo Clinic' of Bispebjerg University Hospital has collected some 400 medical cases of tattoo complications and has additionally researched other groups of tattooed individuals not admitted to the clinic as referenced below. This chapter provides a brief outline of our clinical experience and introduces a descriptive and observational approach, based on clinical pattern recognition, for the diagnosis of medical tattoo reactions. This approach is not a simple translation of diagnosis by histology of tattoo reactions into clinical skin conditions, as is often practised by clinicians. We rely on patient history and clinical signs and symptoms and use histology as a valuable tool for supplementary information and grading of severity prior to treat- 
ment. In our clinic, this treatment is preferably surgery by dermatome shaving, with effective removal of the culprit tattoo pigment in the outer dermis, where it is concentrated. The chapter will not address healing or the phases of recovery following a tattoo, problems, or the issue of aftercare other than what is mentioned in the context of infections.

\section{Definitions of Tattoo Complaints and Complications}

Tattoo complaints concern mild subjectively or objectively abnormal experiences or observations acquired along with and caused by a tattoo. Complaints may address local problems with the tattoo or may be general. Complaints may be acute or subacute and taper out as the tattoo heals. Complaints may also be chronic or chronically intermittent. The tattooed individual in general does not seek medical advice for the complaint.

Tattoo complications are more serious adverse reactions or events after tattooing that manifest as objective abnormalities or pathologies associated with clinical and subjective symptoms of a severity that is considered as a disease or disablement. The tattooed individual with complication in general requests professional medical advice and treatment.

\section{Complaints Associated with Tattoos}

In 2010, Klügl et al. [13] reported health problems associated with tattooed skin based on 3,411 spontaneous reports collected over the internet in German-speaking countries after an open public invitation. In total, $67 \%$ of respondents reported skin problems, and 7\% reported general reactions, which had primarily occurred a few weeks after tattooing. Thus, several hundred complaints were counted. However, the study design did not allow conclusions about the pre- cise incidence or prevalence of complaints and complications.

A Danish study performed in a group of 154 younger persons attending a clinic for sexually transmitted diseases reported tattoo-related complaints of any kind and at any time in $27 \%$, with $58 \%$ of complaints, related to sun exposure [14]. The predominant symptoms were itching/stinging/pain and elevation/inflammation.

Another study, the 'beach study', was conducted at summertime along the coastline of Denmark. Among 144 sunbathers with 146 tattoos, 42 reported complaints of any kind, with $52 \%$ of complaints, related to sun exposure [15].

The studies show that tattoo complaints dominated by itching and swelling are remarkably common and affect approximately $1 / 3$ of tattooed individuals, with 1/5 of tattooed individuals having sun-related problems. Complaints were often reported in dark tattoos, which absorb more light. Skin reactions differed and could be instantaneous, appearing within seconds or minutes after sun exposure, or delayed, appearing within hours and lasting for days. Complaints that were independent of light could last for several weeks and could be constant or intermittent.

\section{Non-Infectious Complications and Adverse Events}

Non-infectious complications can be characterised according to clinical signs and symptoms and according to pathophysiology. Allergic reactions are frequent.

The diagnosis of allergy due to tattoos is performed in the absence of a valid test reference and based on the following clinical criteria:

- Allergic reactions are monomorphic, i.e. uniformly manifested in one particular colour and at all sites where that particular colour is applied in the tattoo

- There is latency of primary sensitisation lasting weeks, months, or years since the 
tattoo was obtained until the reaction

appears

- Once elicited and full blown, the reaction is constant, chronic, cumbersome and refractory to topical corticoid therapy

- A definite criterion for allergy is induction of alike reaction(s) in a hitherto-tolerated tattoo of the same colour located in another anatomical region, i.e. a manifestation of allergic cross-reactivity

In a series of 90 patients with chronic tattoo reactions, an allergy patch test with common allergens, dispersed textile dyes, a battery of risky tattoo inks, and identified culprit inks from individual cases demonstrated that patch test reactions cannot be elicited by application of the potential allergens mentioned [16]. Thus, allergic reactions are not elicited by an allergen directly present in the tattoo ink stock product but rather due to a hapten formed inside the skin over a longer period of time, i.e. months or years. Standard test methods used for cutaneous allergy are consequently not useful in the diagnostic evaluation of suspected allergic reactions. Similarly, in the evaluation of the potential allergy risk of new or known tattoo ink products and ink ingredients, methods such as the Buehler test, the guinea pig maximisation test, and the lymph node assay are inappropriate in rationale and design and are not applicable. These tests are bound to show falsenegative results.

The term 'lichenoid', dating back centuries, to early French dermatology, is sometimes used to describe tattoo reactions with inflammation of the outer skin and epidermal thickening observed by histology. However, the term is imprecise and is used for a broad range of very different skin conditions. The traditional microscopic findings, with lichenoid, granulomatous, and pseudolymphomatous tattoo reactions, does not have a distinct clinical correlate and should not be used to label tattoo reactions as they appear in the clinic. The histologic patterns are not specific and may overlap in the same biopsy.
On clinical and morphologic grounds, reactions and complications can be differentiated into local reaction patterns and general conditions or associations, as described below.

\section{Local Reaction Patterns}

\section{Papulo-Nodular Pattern (fig. 1)}

Tattoos may show papular or nodular thickening and elevation in certain areas, in contrast to other similarly coloured areas of the tattoo that have a normal appearance. Elevations may be round and elongated and may appear confluent, and often appear where the density of pigment is high as a result of 'pigment overload' with injection of too much ink. Elevations are often chronic but may resolve over several months. Papules, which are eroded by scratching, may release their excess pigment and heal, leaving a guttate white spot. The background appears to be an agglomeration of black pigment nanoparticles, i.e. carbon black, forming large bodies in the dermis. The bodies are visible in raw biopsy samples seen by the naked eye. The skin may recognise the bodies as foreign and attempt to expel them via the transepidermal route. However, most material is held back in the dermis by the basement membrane, situated immediately under the epidermis. Papulo-nodular reactions may have other causes, such as needle trauma or instillation of too much ink in the dermis, or more than the skin is able to hold without inflammation. The papulo-nodular pattern is very common and especially noted in black tattoos and black linings. It is not an allergic reaction.

\section{Plaque-Like Pattern (fig. 2)}

These reactions show flat thickening and elevation of the entire tattoo at any site in the tattoo where the problem colour was inserted. Large adherent scales may or may not be present. Inflammation, with lymphocytes, is often major and may extend into the surrounding non-tat- 

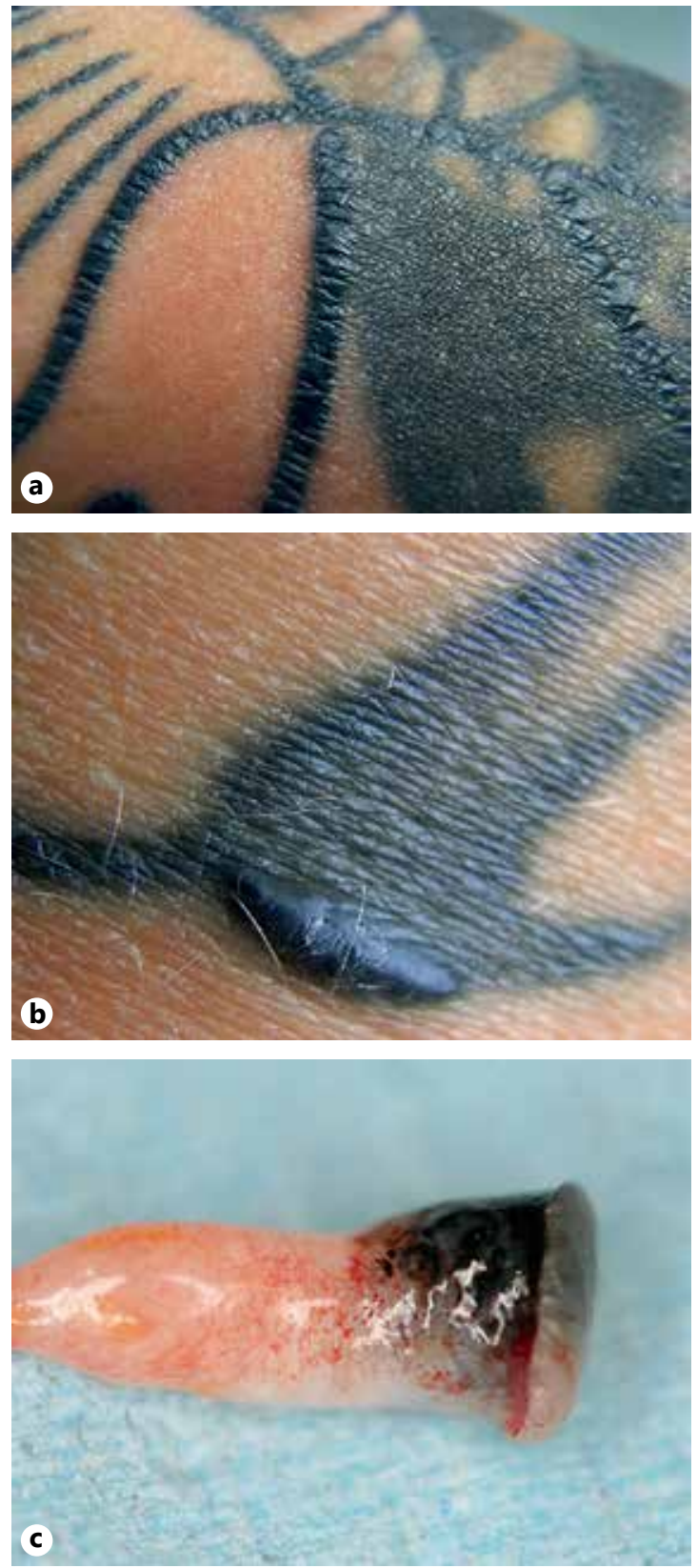

Fig. 1. Papulo-nodular pattern. a Elevated black lines with irregular and confluent thickening, in contrast to a normal black tattoo without any change. b Nodular elevation spontaneously ready to release excess pigment. c Raw punch biopsy of a nodule with visible agglomeration of black pigment particles, which originally were nano-sized.
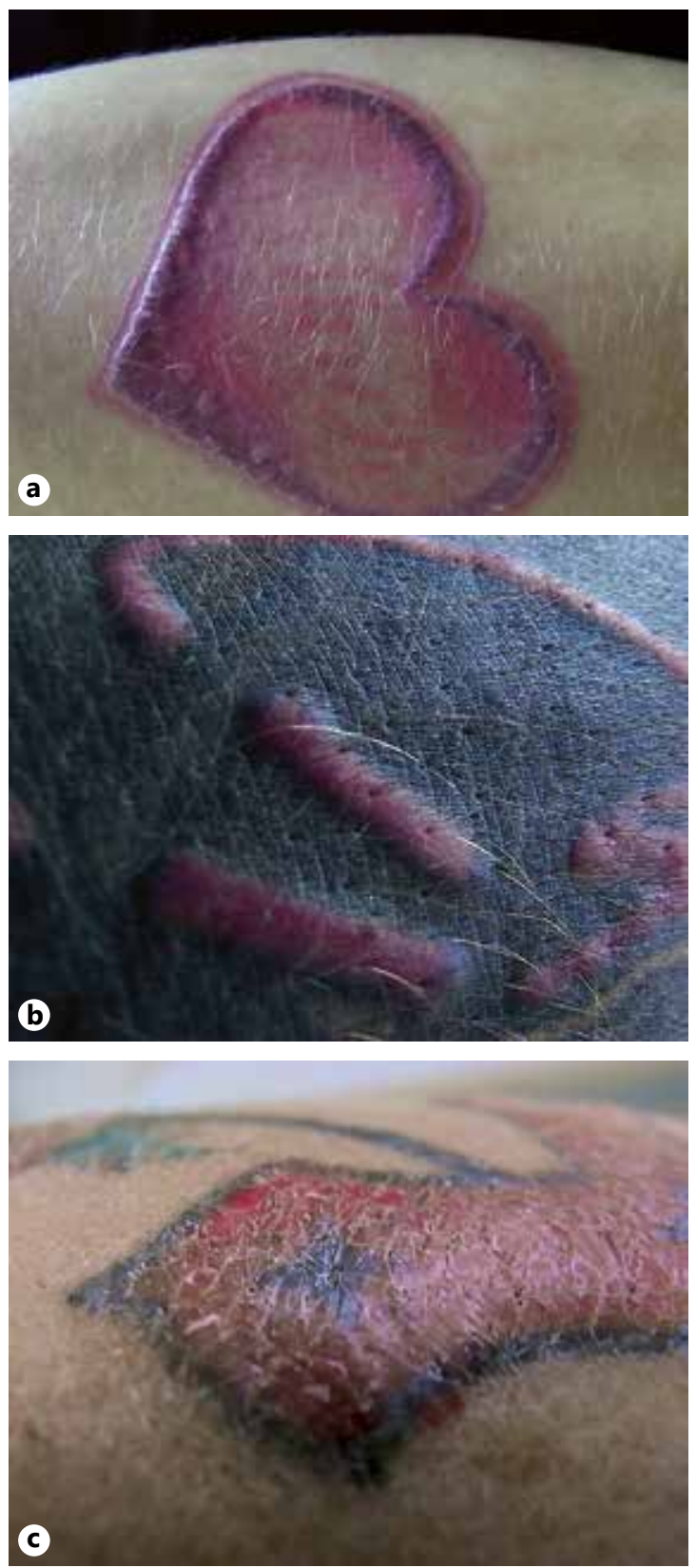

Fig. 2. Plaque-like pattern. a Flat elevation of the entire tattoo where lilac ink was instilled. b Flat elevation of a red tattoo. c Flat elevation and major thickening limited to a red tattoo and sharply demarcated towards normal skin. a-c All have some adherent scaling and minor hyperkeratosis, which traditionally might be labelled 'Iichenoid'. 
tooed skin, and is concentrated in the outer dermis. Histology may show interface dermatitis or any of the traditional histologic patterns, which may overlap in the same biopsy. Epidermal changes with some degree of hyperkeratosis are typical. If the tattoo is a line, such as in tattooed texts, the entire line will be affected. Plaque-like reactions are primarily seen in red tattoos or in nuances of red and are a manifestation of allergy. Green and blue tattoos may also show this pattern as a result of allergy. There may be a visible colour shift from blue to green or from green to blue as the allergic reaction appears.

\section{Excessive Hyperkeratotic Pattern (fig. 3)}

These reactions exhibit inflammation, thickening, and elevation that is major and dominated by massive hyperkeratosis and cornification of the surface, which acquires a structure resembling sandpaper. The entire area dyed with the particular problem colour exhibits the same abnormality. The elevated surface is rather flat, and the pattern might be considered as a plaquelike reaction with an excessive epidermal response to the underlying inflammation. The thickened epidermis may become necrotic and ulcerate. Occasionally, epidermal folding and notches may appear on histology. The notches may go deep and result in epidermal inclusions and fistulas embedded deeply in the dermis. The histologic picture ultimately may be distinct, seen under the microscope as pseudoepitheliomatous hyperplasia. The excessive hyperkeratotic pattern appears in red tattoos and nuances of red. The pattern is considered to indicate allergy, complicated by significant leakage of the basement membrane, escape of the culprit pigment to the epidermis, severe inflammation, and a prominent proliferative response of the epidermis that manifests as excessive hyperkeratosis. In raw punch biopsies, the hyperkeratosis is seen as a whitish layer on top of the underlying dermis, which is colourful due to the red pigment.
Ulcero-Necrotic Pattern (fig. 4)

Aggressive inflammation is directly followed by tissue necrosis and ulceration at any site in the tattoo where the culprit colour was instilled into the skin. Ulceration may affect the full thickness of the dermis and may approach the subcutaneous fat. Necrosis may even extend further into deep tissues if the culprit pigment is disseminated down to the underlying muscle fascia, for example. Necrosis may even affect the regional lymph nodes holding the same pigment. This pattern, especially seen in red tattoos, is a manifestation of a strong allergy. The allergy and haptenisation, seemingly involving tissue components, may progress to autoimmunity, with an attack on non-tattoo sites of the skin integument, including inflammatory reactions, vasculites, bullous reactions, and generally delayed wound healing throughout the skin. The condition may be self-limiting and may heal over a period of several months. Surgical excision is risky and relatively contraindicated.

\section{Lymphopathic Pattern}

Excess pigment particles flow into the lymph and into the lymph vessels to a regional node, where the particles are maintained and may visibly stain the node. Tattoo pigment injected deep into the dermis may also flow into the surrounding skin and produce a visible stain in the direction of the lymph flow. This may result in damage and blockage of distal lymph vessels, with soft tissue swelling and clinically significant lymphoedema. The condition is permanent and shares traits with podoconiosis, a disease among Africans who, via fissures in their bare feet, have microparticles of soil rich in bentonite clay implanted into their tissues. This results in damage and blockage of the lymphatics, eventually resulting in major oedema of the legs, i.e. elephantiasis. Occasionally, weeks after tattooing, tattooed individuals may have swollen and tender regional lymph nodes, although normally without any sequelae other than pigment deposits in the lymph nodes. 


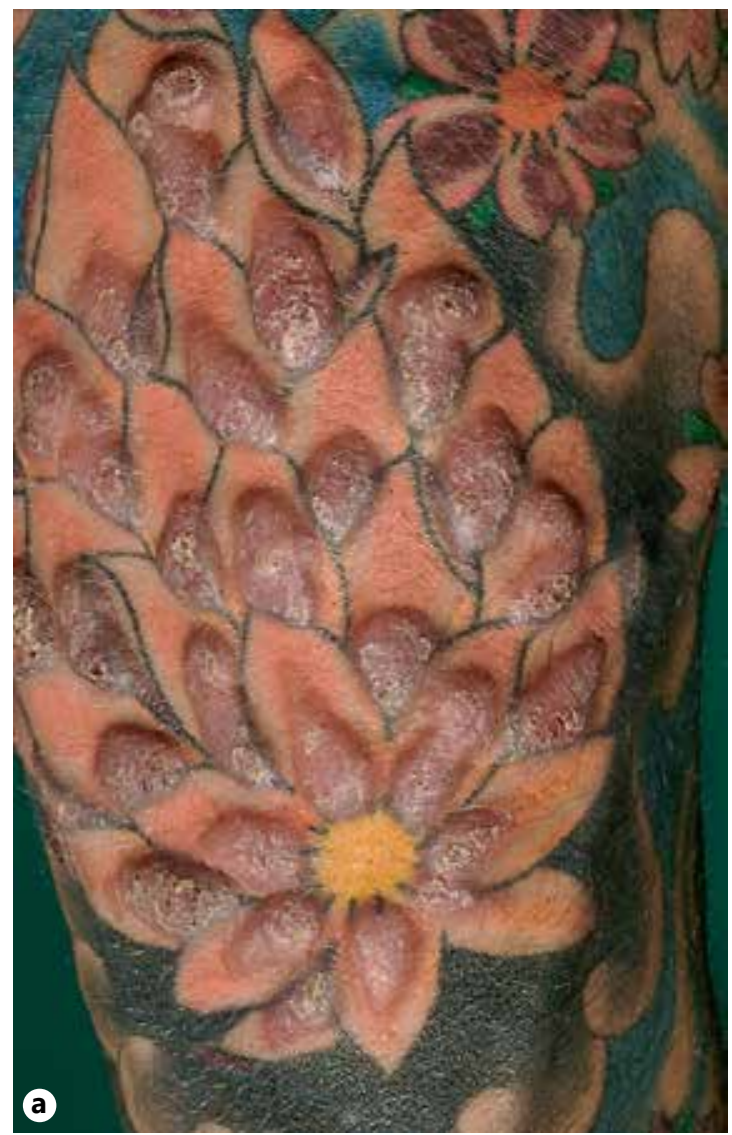

Fig. 3. Excessive hyperkeratotic pattern. a Distinct and prominent thickening at all sites tattooed red, with excessive hyperkeratosis. b Reaction entirely dominated by hyperkeratosis, with a sandpaper-like appearance of the surface, which was flat and exhibits a loss of all skin markings. At a local site, poor nutrition, and ulceration appeared after the hyperkeratosis had loosened. c Detail of excessive hyperkeratotic lesions.
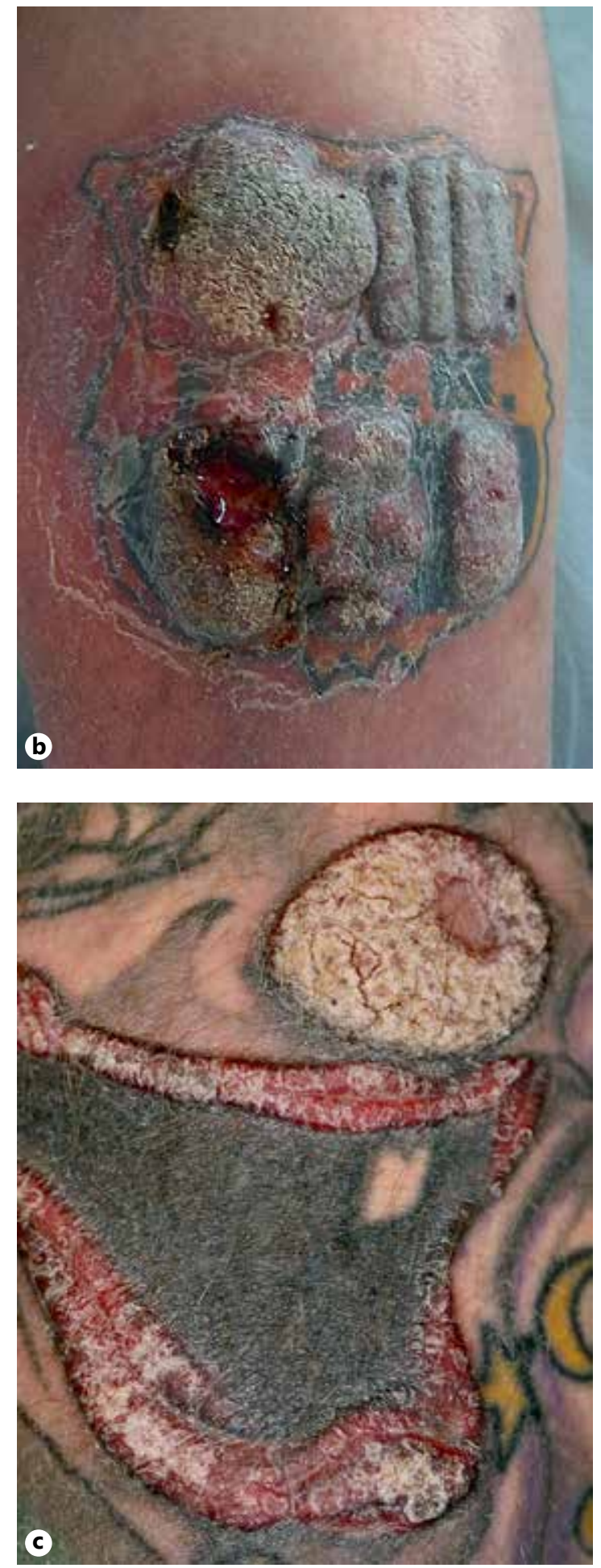


\section{Neuro-Sensory Pattern}

Tattoo reactions and, in particular, allergic reactions often itch a lot and reduce quality of life to a degree comparable to reduction by widespread pruritic skin diseases, even though tattoo reactions typically affect only quite a small area [17]. Tattoos may induce severe and invalidating discomfort, itch and pain, without noticeable clinical changes. The histology of the skin is normal or exhibits minor lymphatic infiltration of the dermis only. It is assumed that chemical(s) in the ink, from the pigment, or released split products may stimulate the $\mathrm{C}$-fibres of the sensory nerves. The sensory change may affect the dermatome or the body regions. Peripheral neuropathy, a herniated vertebral disc, and syringomyelia may be the differential diagnoses. The condition, which may be more frequent in tattoos of the forearm and wrist, has been reported as complex regional pain syndrome [18]. Surgical removal of the tattoo pigment by dermatome may produce radical relief.

\section{Scar Pattern}

Normal tattoos often exhibit mild fibrosis as a sequela of the tattoo needle trauma. Tattooing, with thousands of needle injections at the mid-dermal level, represents massive needle trauma that may result in the development of hypertrophic scars or keloids. Regions such as the chest, the shoulders, and the upper arms are predisposed to abnormal scarring. The risk increases if the needle injections reach the lower dermis and if the tattoo needle is damaged or poor in quality. Electric coil machines appear less risky than rotary machines, which may mill the skin. The risk of inducing a scar obviously depends on the experience and skill of the tattooist. Surprisingly, persons who have prominent keloids for some reason may be tattooed without any abnormal scar formation if the tattooing is performed in the very outer dermis, outside the critical anatomical sites and with care not to traumatise.
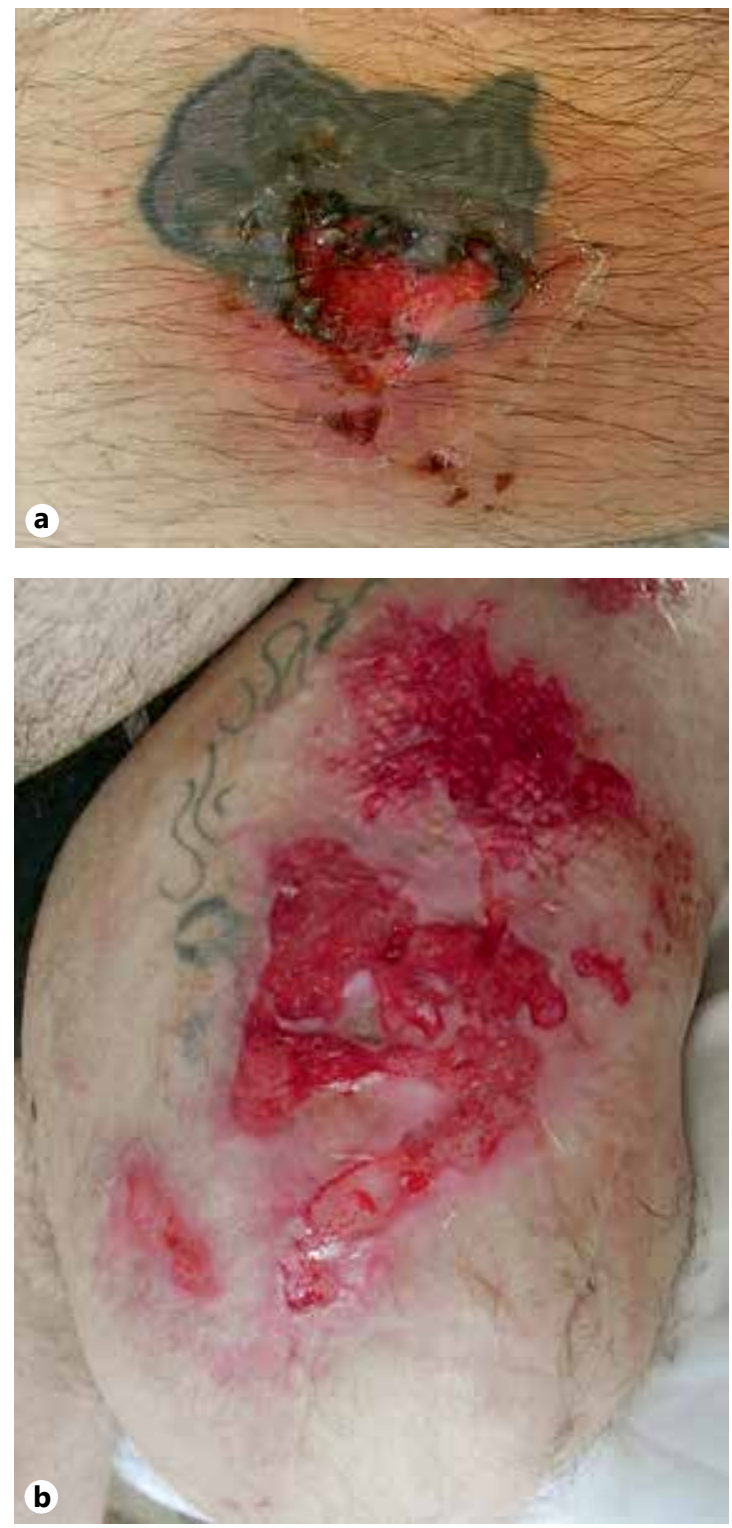

Fig. 4. Ulcero-necrotic pattern. a Ulceration and necrosis exclusively in the red part of a tattoo. The individual had similar necrotic elements in his normal, non-tattooed skin that started as vasculitis-like elements. Healing was spontaneous. b Aggressive ulceration and necrosis in a large tattoo on a leg, necessitating sequential amputations to the femur level. However, a reaction and recurrent ulceration were still present. The non-tattooed skin was also affected. 
General Conditions and Associations

\section{Tattoo-Provoked Illness}

Tattooing may occasionally induce skin afflictions and provoke flares of known skin diseases. The needle trauma of tattooing, with histamine release, routinely produces an urticarial reaction that may be advanced and induce a general flush. Tattooing may be followed by long-lasting urticaria, requiring medical diagnosis and treatment. Eczema, which may be severe and due to a new allergy acquired with the tattoo, such as allergy to paraben preservatives or methylisothiazolinone. The development of allergy to latex proteins originating from the tattooist's gloves and instilled into the skin with the tattoo needle is a serious complication, with a risk of anaphylaxis upon some later exposure to latex. Tattooing is apparently not a clinically relevant inducer of allergy to nickel and chromate VI, despite trace elements of nickel being present in nearly every tattoo ink product.

It is widely unknown whether tattooing and tattoos may trigger or influence other medical diseases or be associated with general diseases specific to tattoos other than autoimmunity, as discussed above. An association with sarcoidosis or, rather, a general sarcoid reaction pattern in the body tissues is known [19].

\section{Existing Skin Disease and Tattoo Reaction}

Urticaria factitia is not congruous with tattooing and may cause major flares and flushing. Atopic skin may also be prone to tattoo-related problems and may more easily become infected since atopic skin often is colonised with staphylococci. Tattooing may also induce local chronic skin diseases such as necrobiosis lipoidica. However, the vast majority of non-infectious skin diseases are not influenced by tattooing. The well-known exception is psoriasis, which may flare up after any mechanical trauma, including a new tattoo, as described by Köbner more than a century ago, generally known as the Köbner phenomenon [20]. Another chapter of this book reviews contraindications for tattooing.

\section{Psycho-Social Disability}

The disparity between tattoo enthusiasts, who proudly wish to display their tattoos to the world, on the one hand and all of those who silently disregard tattoos on the other hand is still very real. The clash may easily reduce job opportunities and end private and professional careers. Many tattooed individuals experience stigmatisation and barriers, especially if tattooed on the face, the neck, or the hands. Many tattoos cannot be removed, even if the best laser technology at the time is applied. Persons who have biker emblems on their skin are in a special class of physical risk. It cannot be denied that social disability can be a consequence of tattooing. The negative impact of an unwanted tattoo on psyche and quality of life is real and a significant complication.

The above shortlist of adverse reaction patterns and conditions associated with tattooing is not exhaustive, and additional manifestations of risks may occur and be depicted in the medical literature.

\section{Infectious Complications}

Tattoo needle penetration of the skin barrier may introduce a range of microorganisms into a person, particularly if the needle is not sterile or is shared among persons, resulting in a range of infections (fig. 5) [21, 22]. Microorganisms may also find their way into the body during the healing phase of a tattoo. Bacterial infections may include not only infections with Staphylococcus, Streptococcus, Pseudomonas, and Clostridium species and even tetanus but also infections with atypical bacteria, such as commensal mycobacteria; tuberculosis; and leprosy. Fungal, parasitic, and spirochaetal (namely, syphilis) in- 

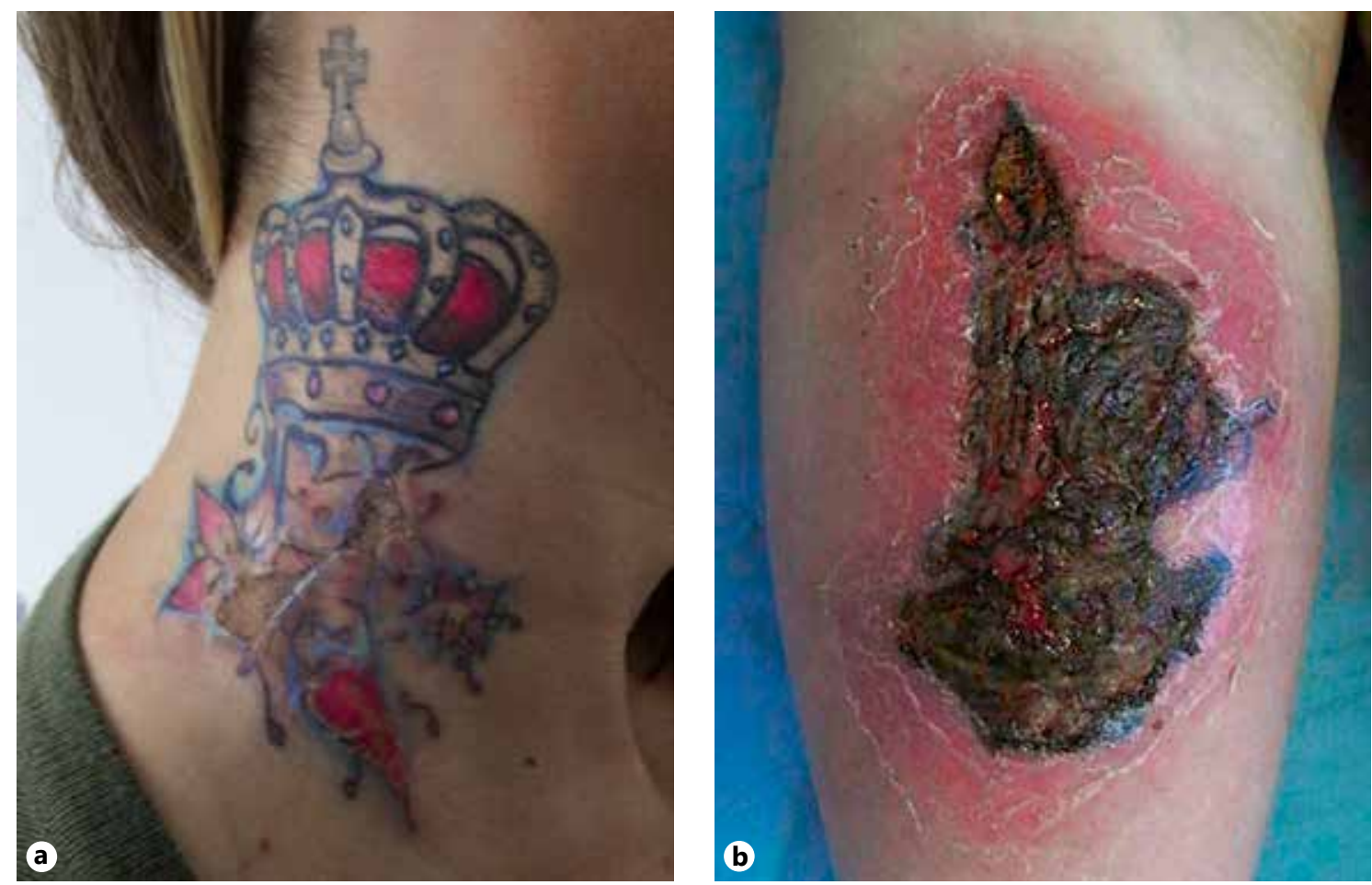

Fig. 5. Infectious complications of tattooing.

a Staphylococcal infection seemingly introduced with yellow tattoo ink. b Pyogenic infection in an entire tattoo, starting in the green part of the tattoo and possibly introduced with the green tattoo ink (for sequential photos, see cover of book). c Bacterial infection of soft tissue of the arm. Patients $\mathbf{a}, \mathbf{b}$, and $\mathbf{c}$ were treated with intravenous antibiotics and recovered without sepsis or other complications.

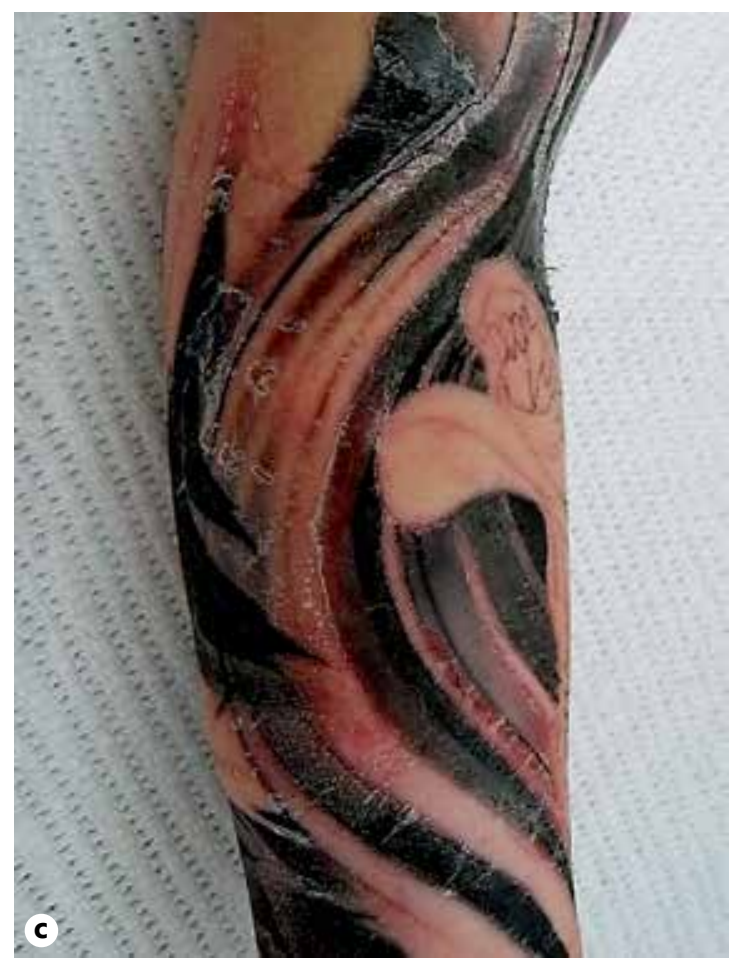


fections may be inoculated. Viral infections include hepatitis B and C; human immunodeficiency virus (HIV); herpes; and viruses causing local infection, such as warts, molluscum, and condylomata.

Infective microorganisms may originate from the tattooed person; the parlour; the tattooist; the needle or the instruments; or, last but not least, the tattoo ink stock product or contaminated water used by the tattooist for dilution of the ink. A recent study showed that, in accordance with other studies, as many as $10 \%$ of new inks were contaminated with bacteria that are pathogenic to humans and the label 'Sterile' on a product was not reliable [10]. The spontaneous resistance of tattooed individuals to infectants introduced by a tattoo needle must be high. Most tattooed individuals are young and in good health. Fortunately, we have not observed a very high incidence of bacterial infections following tattooing, although precise data on the incidence and prevalence of infection related to tattooing are not available. Persons having weaknesses and impaired immune systems must be especially vulnerable. Some infections are rare, endemic, or historical, albeit still being a potential treat.

Exposure of the population to pathogenic microorganisms is dynamic and may always change over time. There is presently and will continue to be a special threat from staphylococci resistant to antibiotics and bacteria, Escherichia coli with acquired transfer resistance carried by DNA segments, or extended-spectrum beta-lactamase resistance. There is a group of bacteria, 'flesh-eating bacteria', which cause deep wounds and necrotising fasciitis. The infection is life-threatening and may require acute surgery, with wide excision and even amputation. Aggressive strains of E. coli from food may cause severe casualties and death, as recently seen in Germany, and E. coli can be found in new tattoo inks. Bulk contaminated tattoo inks represent a hitherto and unforseen major potential risk for spread epidemic infection across national borders.
In today's clinic, pyogenic infections with staphylococci, streptococci, Pseudomonas, and E. coli and transfer of hepatitis B and C are the main risks associated with tattooing practices.

Infections may be superficial and may manifest as impetigo and pyogenic infection. Abscess formation may develop and require surgical incision. Profound infections may present as erysipelas or as soft-tissue infection, i.e. cellulitis, with swelling of the distal or the entire limb. Oral or intravenous antibiotic treatment is required. Bacteria may pass into the bloodstream and cause sepsis, with high fever, poor general condition, affliction and impairment of vital organs, and ultimately death, unless antibiotic treatment is given and effective in the particular case. Exceptional cases of death from tattooing occur even today. Bacteria from tattooing may also enter the blood, infect the heart valves, and result in cardiac insufficiency. There is clearly a need for early and efficient treatment of bacterial infection, if possible after isolation of the causative microorganism, which also requires culture from the culprit ink. The need for tracing the route of infection to the origin is often neglected. Proper treatment also includes prevention of new cases infected by the same source.

Mycobacterial infections develop slowly. They are often caused by contaminated tap water used for the dilution of ink. Such infections are quite rare but need special diagnostic sampling and treatment. Mycobacterial infections typically develop into local outbreaks in the neighbourhood of a parlour.

From a preventive point of view, visiting 'star' tattooists, who travel around with their tools and inks, presents a special risk of spread of infection, just as tattooing at festivals and conventions may do.

The threat of hepatitis virus $\mathrm{B}$ and $\mathrm{C}$ remains. In contrast to HIV, these viruses require only a small inoculum to infect a person. Hepatitis remains frequent among drug users. Tat- 
tooists' use of gloves and disposable needles helped to reduce this risk. Hepatitis due to tattooing is easily overlooked since this infection may develop slowly and may go unnoticed and since hepatic failure may take decennia to develop. HIV transferred by tattooing may not be noticed and linked to a tattoo since the person may have other risk exposures related to various sexual contacts.

\section{Life-Threatening Hazards}

In the past, it was recognised that infections introduced with a tattoo could kill the tattooed individual. The French marine was aware of amputations and death among the mariners and prohibited the mariners from being tattooed [23]. Today death from tattooing is exceptional albeit still a potential risk. As mentioned above the risk may vary over time and abruptly become real. Tetanus infection is today very rare, and populations normally are vaccinated.
Allergic reactions of tattoos are mostly delayed and do not threaten or shorten life unless the allergy is strong, necrotising, or associated with autoimmunity affecting normal tissues.

Syncope while being tattooed may potentially occur due to pain, epilepsy, or cardiac dysfunction or arrest, therefore, the tattooist must be aware of such risk and not tattoo predisposed persons.

There is a rare and special risk associated with allergy to latex protein. Latex particles from the tattooist's gloves can elicit anaphylactic shock in the already-sensitised consumer right as the session starts. During tattooing, tattooists can introduce multiple latex particles from the gloves into the skin and initiate allergic sensitisation of the customer, who, upon some later latex exposure, may unexpectedly and suddenly enter a state of life-threatening anaphylaxis. Some fruits can cross-react with latex and provoke anaphylaxis. Latex gloves also may provoke delayed type allergy causing hand eczema as an occupational disease affecting the tattooist.

\section{References}

1 De Cuyoer C, Perez-Cotapos M-L: Dermatologic Complications with Body Art. Tattoos, Piercings and Permanent Make-Up. Berlin-Heidelberg, Springer Verlag, 2010.

$\checkmark 2$ Goldstein N: Tattoos. J Dermatol Surg Oncol 1979;5:846-916.

3 Jacob CI: Tattoo-associated dermatoses: a case report and review of the literature. Am Soc Derm Surg 2002;28:962965.

4 Kazandjieva J, Tsankov N: Tattoos: dermatological complications. Clin Dermatol 2007;25:375-382.

5 Kaatz M, Elsner P, Bauer A: Body-modifying concepts and dermatologic problems. Clin Dermatol 2008;26:35-44.

6 Mataix J, Silvestre JF: Cutaneous adverse reactions to tattoos and piercings. Acta Dermosifilogr 2009;100:643-656.
7 Kluger N: Cutaneous complications related to permanent decorative tattooing. Expert Rev Clin Immunonol 2010;6: 363-371.

8 Desai NA, Smith ML: Body art in adolescents: paint, piercings and perils. Adolesc Med 2011;22:97-118.

$\checkmark 9$ Wenzel SM, Rittman I, Landthaler M, Bäumler W: Adverse reactions after tattooing: review of the literature and comparison to results of a survey. Dermatology 2013;226:138-147.

$>10$ Høgsberg T, Saunte DM, FrimodtMøller N, Serup J: Microbial status and product labelling of 58 original tattoo inks. J Eur Acad Dermatol Venereol 2013;27:73-80.

1 Baumgartner A, Gautsch S: Hygienicmicrobiological quality of tattoo- and permanent make-up colours. J Verbr Lebensm 2011;6:319-325.
12 Kluger N, Koljonen V: Tattoos, inks and cancer. Lancet Oncol 2012;13:e161e168.

13 Klügl I, Hiller K-A, Landthaler M, Bäumler W: Incidence of health problems associated with tattooed skin: a nation-wide survey in German-speaking countries. Dermatology 2010;221:43-50.

14 Høgsberg T, Carlsen KH, Serup J: High prevalence of minor symptoms in tattooos among a young population tattooed with carbon black and organic pigments. J Eur Acad Dermatol Venereol 2013;27:846-852.

15 Hutton Carlsen K, Serup J: Photosensitivity and photodynamic events in balck, red and blue tattoos are common: a 'Beach Study'. J Eur Acad Dermatol Venereol 2013, DOI: $10.1111 /$ jdv.12093. 
16 Serup J, Hutton Carlsen K: Patch test study of 90 patients with tattoo reactions: negative outcome of allergy patch to baseline batteries and culprit inks suggests allergen(s) are generated in the skin through haptinization. Contact Dermatitis 2014;71:255-263.

17 Hutton Carlsen K, Serup J: Patients with tattoo reactions have reduced quality of life and suffer from itch: Dermatology Life Quality Index and Itch Severity Score measurements. Skin Res Technol 2014, DOI: 10.1111/srt. 12164.
18 Morte PD, Mgee LM: Hyperalgesia after volar wrist tattoo: a case of complex regional pain syndrome? J Clin Neuromuscular Dis 2011;12:118-121.

19 Morales-Calaghan AM, Aguilar-Bernier M, Martinez-Garcia G, Miranda-Romero A: Sarcoid granuloma on black tattoo. J Am Acad Dermatol 2006;55:71-73.
20 Köbner H: Zur aethiologie der psoriasis. Viertel Jahresschrift für Dermatologie und Syphilis 1876;3:559-561.

21 Long GE, Rickman LS: Infectious complications of tattoos. Clin Infect Dis 1994;18:610-619.

22 Kluger N: Complications infectiouses cutanées assciées au tatouage permanent. Médicine et Maladies Infectieuses 2011;41:115-122.

23 Berchon E: Histoire Médicale du Tatouage. Paris, J-B Bailliè et Fils, 1869.

Prof. Jørgen Serup, MD, DMSc

Dermatology D41, Bispebjerg University Hospital

Bispebjerg Bakke 23

DK-2400 Copenhagen NV (Denmark)

E-Mail joergen.vedelskov.serup@regionh.dk 